\title{
Conservative Management of Placenta Accreta of Seven Cases
}

\author{
Bouchra Fakhir*, Mouna Zaki, Karam Harou, Ahlam Bassir, Lahcen Boukhan, \\ Yasser Aitbenkeddour, Hamid Asmouki, Abderraouf Soummani \\ Gynecology Obstetrics Department, University Hospital Mohammed VI, Cadi Ayyad University, Marrakesh, Morocco \\ Email: *fakhirb@gmail.com
}

How to cite this paper: Fakhir, B., Zaki, M., Harou, K., Bassir, A., Boukhan, L., Aitbenkeddour, Y., Asmouki, H. and Soummani, A. (2018) Conservative Management of Placenta Accreta of Seven Cases. Open Journal of Obstetrics and Gynecology, 8, 660-668.

https://doi.org/10.4236/ojog.2018.87070

Received: February 21, 2018

Accepted: June 24, 2018

Published: June 27, 2018

Copyright $\odot 2018$ by authors and Scientific Research Publishing Inc. This work is licensed under the Creative Commons Attribution International License (CC BY 4.0).

http://creativecommons.org/licenses/by/4.0/

\begin{abstract}
Introduction: Placenta accreta is a potentially life threatening obstetrical condition. The incidence has increased. Diagnosis before delivery allows multidisciplinary planning in an attempt to minimize potential maternal or neonatal morbidity and mortality. Prenatal ultrasonography is used to support the diagnosis and guide clinical management leading probably to favorable outcomes. Actually a conservative option which includes leaving all or part of the placenta in situ when fertility preservation is desired is recommended. Methods: We retrospectively reviewed the medical records of all patients diagnosed with placenta accreta in gynecology-obstetrics department of the university hospital Mohammed the VI of Marrakesh; Morocco; from January the first 2014 to January the second 2016. Results: We found seven cases. We described: The epidemiological characteristics, risk factors, management of placenta accreta, outcomes and prognosis. The incidence of placenta accreta was $1 / 3847$ deliveries. The mean term of delivery was 35 weeks. We have adopted a successful conservative treatment in six cases (71.4\%). The radical treatment was adopted in one patient initially admitted for severe post-partum hemorrhage; the prognosis was good in $85.7 \%$ cases. Conclusion: Conservative management of placenta accreta is a safe and efficient and is an interesting alternative for hysterectomy.
\end{abstract}

\section{Keywords}

Placenta Accreta, Conservative Management, Scared Uterus, Postpartum Bleeding, Hysterectomy, Placenta Previa

\section{Introduction}

Placenta accreta spectrum or abnormally invasive placenta (AIP) is an obstetrical complication associated with significant maternal morbidity and mortality. It 
is caused by a defect in the decidua basalis resulting in an abnormally invasive placental implantation [1]. This disruption is often related to previous uterine scars, including $\mathrm{C}$-sections and prior uterine curettage. The other risk factors associated with placenta accreta are multiparity; placenta previa; prior intrauterine infections; and maternal age over 35 years [2] [3] [4].

Histologically, placenta accreta is identified by trophoblastic invasion of the myometrium in the absence of intervening deciduas [1]. The spectrum includes invasion of the superficial myometrium (accreta), invasion into deeper myometrial layers (increta), and invasion through the serosa and/or adjacent pelvic organs (percreta) [5].

Traditionally, caesarean hysterectomy at the time of delivery has been the favorite management strategy for placenta accreta prenatally diagnosed. Many cases are identified only at the time of delivery when forcible attempts at manual removal of the placenta are unsuccessful [6]. This situation increases emergency hysterectomy as well. Conservative management by leaving the placenta inside the uterus after birth was successfully performed in many publications; it is a useful strategy when hysterectomy appears to be difficult to perform (placenta percreta) or when fertility preservation is considered [6] [7].

The aim of this study is to describe the successful experience of our medical center with the conservative management of this particular problem.

\section{Methods}

We retrospectively reviewed the medical records of all patients diagnosed with placenta accreta in gynecology-obstetrics department of the university hospital Mohammed the VI of Marrakesh; Morocco; from January the first 2014 to January the second 2016. We included all the patients who were diagnosed with placenta accreta prenatally or even during delivery; and who underwent a conservative management with placenta left insitu as first management. We describedepidemiological characteristics, risk factors, management of placenta accreta, and patient's outcome.

This study is a descriptive one, it has limits regarding patient's inclusion because placenta accreta is under-diagnosed and many severe post partum hemorrhage (PPH) could be treated without having the exact diagnosis of placenta accreta.

Oral patient's approval was asked for the use of pictures; in our institution no need of ethics committee approval for this type of studies.

Calculations was conduct using simple excel 2013 system.

\section{Results}

During the study period, seven cases were found. The incidence of placenta accreta was $1 / 3847$ deliveries in our hospital $(0.02 \%)$. The mean age was 31 years and ranged from 23 to 40 years, the average parity was 3 . Median gestational age was 33 weeks ranged. Risk factor for placenta accreta was history of c-section de- 
livery in all cases, three women had more than one C-section and four had only one. One of them also had a previous curettage. The other risk factors were maternal age greater than 35 years old $42.8 \%$, multiparity $71.4 \%$ (Table 1 ).

The diagnosis was established by ultrasound in five cases $(71.42 \%)$, features of placenta accreta included vascular spaces within the placenta, thinning of the myometrium overlying the placenta, loss of the retroplacental "clear space", increased vascularity of the uterine serosa and turbulent blood flow through the lacunae on Doppler ultrasonography (Figures 1-3). Two patients had magnetic resonance imaging to confirm the diagnosis after ultrasonound (Figure 4).

In two cases, placenta acreta was discovered during a caesarian section, indicated for hemorrhagic placenta previa.

The timing for delivery ranged from 31 to 38 weeks, depending on the moment of patient arrival in our medical center and the presence of antepartum bleeding; For that, corticosteroid administration was necessary before 34 weeks to improve neonatal outcomes.

Conservative treatment was performed in six cases (85.7\%), a midline cutaneous incision with a vertical uterine incision far from the placental bed was performed. After the new born was delivered, the cord was cut at the insertion site and the uterine cavity was closed with placenta intactleft inside. No attempt was made to remove the placenta. (Figure 5)

A hysterectomy was performed in one patient for shock and hemorrhageafter immediate failure of the conservative treatment.

One of the six patients had secondary hysterectomy for bleeding relapse in day 1.

Postoperative prophylactic antibiotic therapy: Amoxicillin and clavulanic acid was systematically administered for 10 days to minimize the risk of infection

Uterine artery embolization is not available in our medical center so the conservative treatment was combined with bilateral hypogastric ligation in four cases $(66.6 \%)$ to minimize blood loss. One dose of $1 \mathrm{mg} / \mathrm{m}^{2}$ of Methotrexatewas administrated intramuscularly as an adjuvant treatment for the 6 cases. Two patients had received Methotrexate twice, because of a recurrence of bleeding at day 6 after c-section, which caused us to avoid a secondary hysterectomy.

Patients were followed weekly with ultrasound Doppler, serum b-hCG and blood infection indicators (Figure 6 and Figure 7). The prognosis was good in six cases (85.7\%). The patient who underwent cesarean hysterectomy died with disseminated intravascular coagulation in uncontrolled preeclampsia conditions. Three months' later patients have had almost normal ultrasound findings and b-hCG value of less than $5 \mathrm{mIU} / \mathrm{ml}$. In the five cases placenta resorption was observed, no need to any dilation-curettage, no placenta expulsion was observed, no further complication was observed.

\section{Discussion}

The incidence of placenta accreta is increasing largely due to the global increase in $\mathrm{C}$-section deliveries, it approximates 1/500 deliveries and $\mathrm{Wu}$ et al. found an 
Table 1. Results.

\begin{tabular}{|c|c|c|}
\hline & Number (\%) & mean \\
\hline Age (years-old) & & $31.5(23$ to 40$)$ \\
\hline Parity & & III P \\
\hline Gestational age (weeks) & & 33 (31 to 38$)$ \\
\hline \multicolumn{3}{|l|}{ Risk factors: } \\
\hline Scared uterus & $4(57.5)$ & \\
\hline Previous curettage & $2(28.5)$ & \\
\hline Double scared uterus & $1(14.2)$ & \\
\hline Triple scared uterus & $1(14.2)$ & \\
\hline \multicolumn{3}{|l|}{ Diagnosis of placenta accreta: } \\
\hline Ultrasonography & $5(71.4)$ & \\
\hline Magnetic resonance imaging & $2(28.5)$ & \\
\hline
\end{tabular}

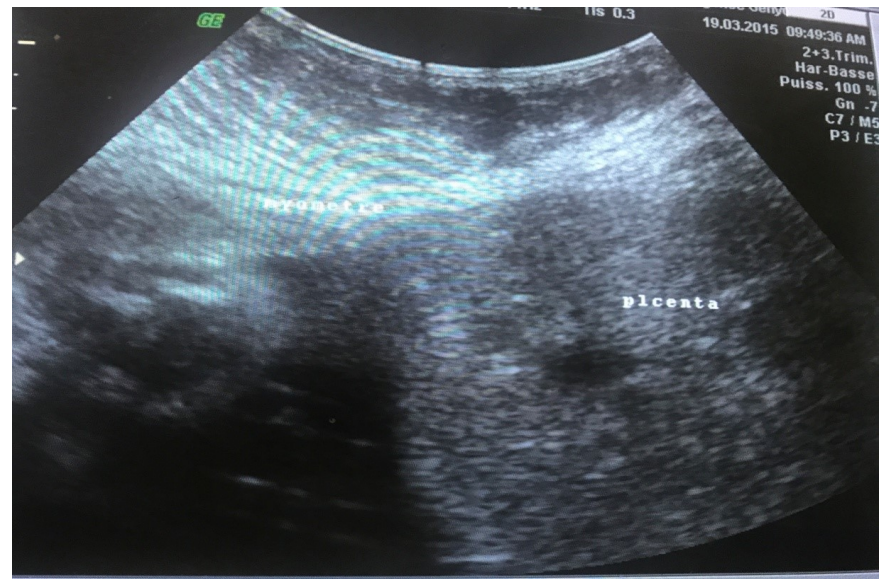

Figure 1. Antenatal diagnosis of placenta accreta (intraplacental lacunae).

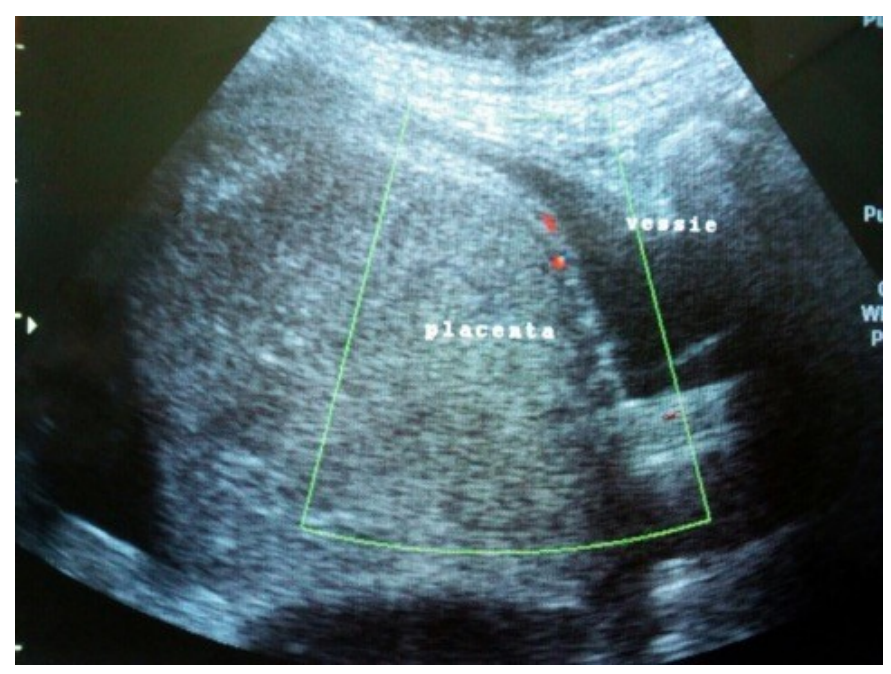

Figure 2. Thinning of the myometrium overlying the placenta, loss of the retroplacental "clear space". 


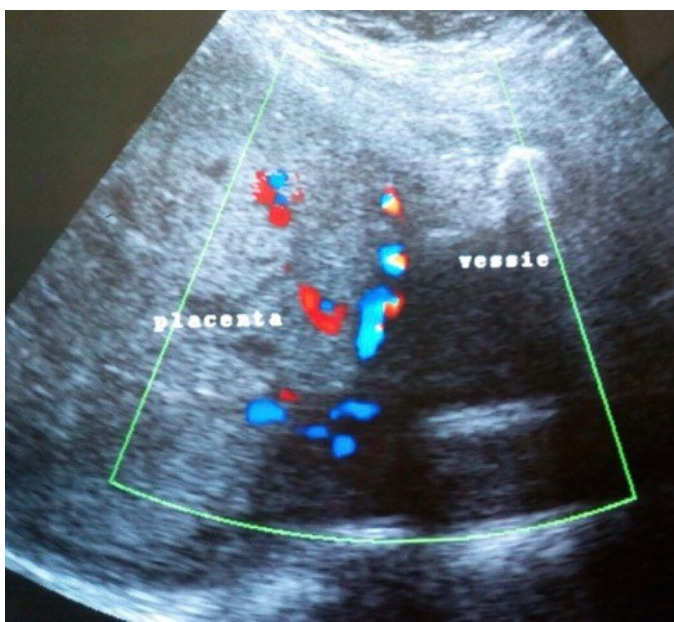

Figure 3. Increased vascularity of the uterine serosa and turbulent blood flow through the lacunae on doppler ultrasonography.

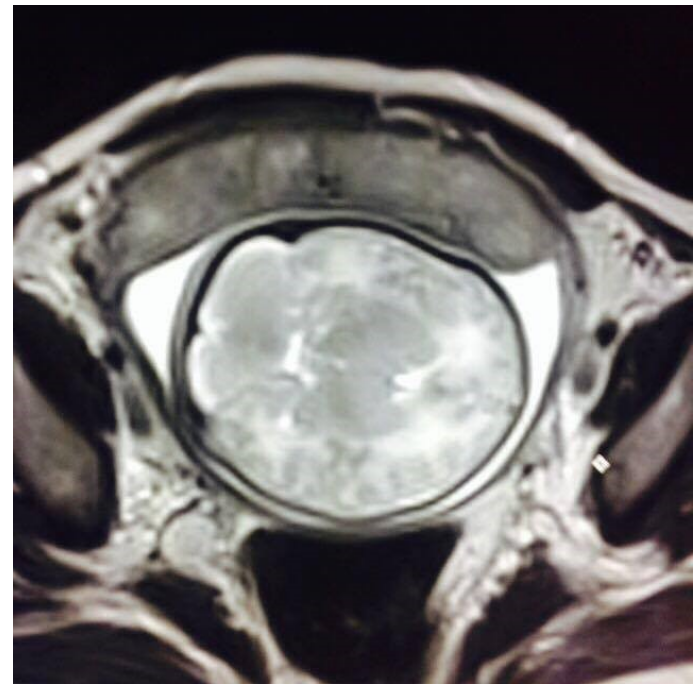

Figure 4. Placenta accreta in MRI.

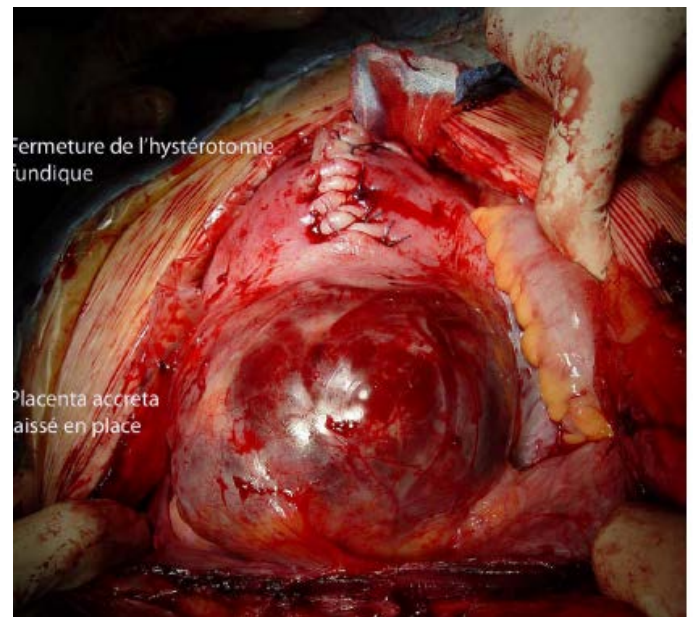

Figure 5. Surgical conservative management. 


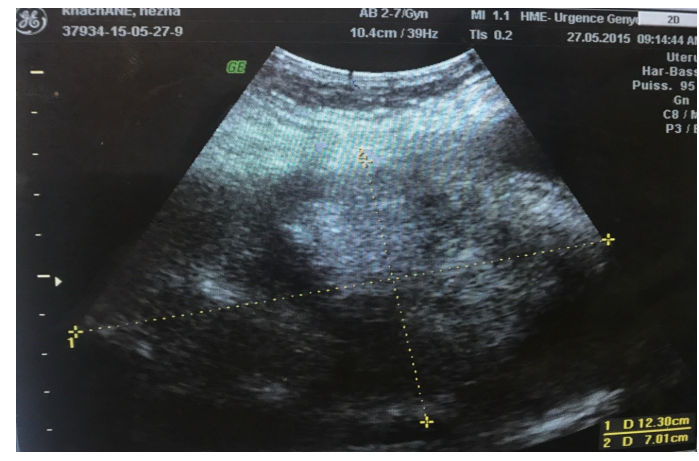

Figure 6. Ultrasonography 15 days after surgery.

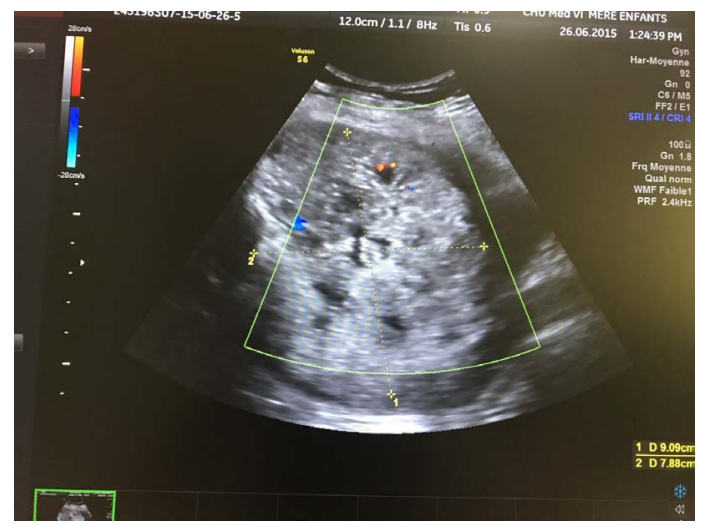

Figure 7. Ultrasonography 1 month later.

incidence of $1 / 533$ pregnancies in their center [3]. We found a lower incidence of $1 / 3847$ deliveries because of the lack of prenatal diagnosis and the incomplete data of histology analysis of the PPH hysterectomy specimens.The risk of developing placenta accreta increases with the number of previous cesarean deliveries. It ranges from $39 \%$ to $60 \%$ of placenta preavia accompanied with two or more prior c-section deliveries [8] [9]. Alanis et al. reviewed 72 cases of placenta accreta that were treated conservatively, among $15 \%$ of women who had a subsequent pregnancy, $18 \%$ developed a repeated placenta accreta [10] [11]. Prenatal diagnosis of placental invasion is necessary and may be lifesaving. Ultrasound is the first line imaging modality; however, in equivocal cases, pelvic MRI is recommended particularly when dealing with cases of posterior placenta [12] [13]. The advantage of prenatal diagnosis is to plan the delivery in a suitable center with a multidisciplinary team and adequate equipment and resources including a maternity-oriented intensive care unit (ICU) [12] [13]. Ultrasonographic features of placenta accreta include vascular spaces within the placenta, thinning of the myometrium overlying the placenta, loss of the retroplacental "clearspace", protrusion of the placenta into the bladder, increased vascularity of the uterine serosa and turbulent blood flow through the lacunae on doppler ultrasonography [13]. The optimal timing of delivery for the patients with placenta accreta remains controversial. Whatever the gestational age chosen, practitioners have to plan an organization to include the unexpected occurrence 
of acute bleeding requiring an emergency c-section [14]. In a large multicenter trial, $23.9 \%$ of women with placenta accreta in whom cesarean section was planned had an emergency delivery [15]. O’Brien et al. reported that after 35 weeks, $93 \%$ of patients with placenta accreta experienced hemorrhage necessitating delivery [8] [16]. An analytical decision model showed that the preferred gestational age of delivery is at or beyond 34 weeks, but not later than 37 weeks [15]. Traditionally, caesarean hysterectomy at the time of delivery has been the preferred management strategy for placenta accreta. Not only this approach precludes future fertility, but it also exposes to intraoperative bleeding risks regarding surgical difficulties in percreta situation [17]. For women who wish to conserve their reproductive function, other options have been described, extirpative approach with attempt to remove the placenta and conservative managementwith or without partial resection of placenta.A recent Turkish paper comparing retrospectivally hysterectomy without placenta removal (G1); placenta left in situ (G2) and placenta removal with conservative surgery (G3) concluded that the blood loss and the amount of blood products transfusion were the lowest in G2 and uterine preservation rate was similar in G2 and G3 with no difference in the three groups in surgical complications [18]. The American college of obstetricians and gynecologists recommended management of suspected placenta accreta is planned preterm cesarean hysterectomy with the placenta left in situ ;planned attempts of placenta removing should be avoided, but if fertility needs to be preserved a conservative managment with placenta left in situ should be considered after patient counseling about this approch complications [8].

Pre or post-operative embolization of uterine arteries may help to prevent excessive intra-operative and immediat post operative blood loss and to promote placental resorption [19]. Successfully some cases of placenta percreta managed conservatively using uterine artery embolisation were reported with clinical success at around 86\% [20]. In our serie, bilateral hypogastric artery ligation was performed in four cases, even if systematic recourse of this procedure is not recommended [8] [21]. because uterine artery embolisation is not available in our medical centre.

Adjuvant therapy with methotrexate has been used to expedite the placenta resorption [22] [23]. Its use as an auxiliary treatment to reduce placental mass is not well proven to date [8] [10]. In our study, all patients were treated with intramuscular injection of methotrexate immediately in postpartum, and one of them have had a second injection after a bleeding relaps at day 6 with successful maintaining of conservative management. It has been hypothesized that methotrexate acts by inducing placental necrosis and expediting a more rapid involution of the placenta [10]. In our study, conservative treatment failed in one case (16.6\%) and led to hysterectomy. Endometritis is frequently reported as complication of conservative treatment. In this way, prophylactic antibiotics may be helpful in the immediate postpartum period to reduce this risk [16]. All our pa- 
tients was treated by antibiotics and no serious infection complications was reported.

\section{Conclusion}

Conservative management with placenta left in situ in stable patients appears to be a safe alternative to extirpative management. It facilitates the avoidance of hysterectomy and preserves fertility. Bilateral hypogastric artery ligature and methotrexate adjuvant treatment may help in the successfully achievement of this strategy. Antenatal diagnosis should be improved among patients with a high risk profile for placenta accreta using ultrasonography and in selected cases by MRI in order to optimize conservative strategy.

\section{References}

[1] Oyelese, Y. and Smulian, J.C. (2006) Placenta Previa, Placenta Accreta, and Vasa Previa. Obstetrics and Gynecology, 107, 927-941. https://doi.org/10.1097/01.AOG.0000207559.15715.98

[2] Miller, D.A. Chollet, J.A. and Goodwin, T.M. (1997) Clinical Risk Factors for Placenta Previa-Placenta Accreta. American Journal of Obstetrics \& Gynecology, 177, 210-214. https://doi.org/10.1016/S0002-9378(97)70463-0

[3] Wu, S., Kocherginsky, M. and Hibbard, J.U. (2005) Abnormal Placentation: Twenty-Year Analysis. American Journal of Obstetrics \& Gynecology, 192, 1458-1461. https://doi.org/10.1016/j.ajog.2004.12.074

[4] Zelop, C. Nadel, A., Frigoletto Jr., F.D., Pauker, S., MacMillan, M. and Benacerraf, B.R. (1992) Placenta Accreta/Percreta/Increta: A Cause of Elevated Maternal Serum Alpha-Fetoprotein. Obstetrics and Gynecology, 80, 693-694.

[5] Tong, S.Y.P., Tay, K.H. and Kwek, Y.C.K. (2008) Conservative Management of Placenta Accreta: Review of Three Cases. Singapore Medical Journal, 49, e156-e159.

[6] Timmermans, S., Van Hof, A.C. and Duvekot, J.J. (2007) Conservative Management of Abnormally Invasive Placentation. Obstetrical and Gynecological Survey, 62, 529-539. https://doi.org/10.1097/01.ogx.0000271133.27011.05

[7] Sentilhes, L., Ambroselli, C., Kayem, G., et al. (2010) Maretnal Outcome after Conservative Treatment of Placenta Accreta. Obstetrics \& Gynecology, 115, 526-534. https://doi.org/10.1097/AOG.0b013e3181d066d4

[8] The American College of Obstetricians and Gynecologists. Committee Opinion N: 529 July 2012; Reaffirmed 2017.

[9] Miller, D.A., Chollet, J.A. and Goodwin, T.M. (1997) Clinical Risk Factors for Placenta Previa-Placenta Accreta. American Journal of Obstetrics and Gynecology, 177, 210-214. https://doi.org/10.1016/S0002-9378(97)70463-0

[10] Lin, K., Qin, J., Xu, K., Hu, W. and Lin, J. (2015) Methotrexate Management for Placenta Accreta: A Prospective Study. Archives of Gynecology and Obstetrics, 291, 1259-1264. https://doi.org/10.1007/s00404-014-3573-1

[11] Nunes, C., Carvalho, R.M., et al. (2014) Diagnosis of Placenta Accreta by Ultrasonography: A Gold Standard? Acta Obstetrica e Ginecologica Portuguesa, 8, 136-140.

[12] Derman, A.Y., Nikac, V., Haberman, S., et al. (2011) MRI of Placenta Accreta: A New Imaging Perspective. $A J R, 197,1514-1521$.

[13] Comstock, C.H. (2005) Antenatal Diagnosis of Placenta Accreta: A Review. Ultra- 
sound in Obstetrics and Gynecology, 26, 89-96. https://doi.org/10.1002/uog.1926

[14] Robinson, B.K. and Grobman, W.A. (2010) Effectiveness of Timing Strategies for Delivery of Individuals with Placenta Previa and Accreta. Obstetrics \& Gynecology, 116, 835-842. https://doi.org/10.1097/AOG.0b013e3181f3588d

[15] Levine, A.B., Kuhlman, K. and Bonn, J. (1999) Placenta Accreta Comparison of Cases Managed with and without Pelvic Artery Balloon Catheters. The Journal of Maternal-Fetal Medicine, 8, 173-176. https://doi.org/10.1002/(SICI)1520-6661(199907/08)8:4<173::AID-MFM7>3.0.CO;2 $-\mathrm{V}$

[16] O’Brien, J.M., Barton, J.R. and Donaldson, E.S. (1996d) The Management of Placenta Percreta: Conservative and Operative Strategies. American Journal of Obstetrics \& Gynecology, 175, 1632-1637. https://doi.org/10.1016/S0002-9378(96)70117-5

[17] Kayem, G. and Keita, H. (2014) Management of Placenta Previa and Accrete. European Journal of Obstetrics \& Gynecology and Reproductive Biology, 43, 1142-1160. https://doi.org/10.1016/j.jgyn.2014.10.007

[18] Kutuk, M.S., Ak, M. and Ozqun, M.T. (2018) Leaving the Placenta in Situ versus Conservative and Radical Surgery in the Treatment of Placenta Accreta Spectrum Disorders. International Journal of Gynecology \& Obstetrics, 140, 338-344. https://doi.org/10.1002/ijgo.12308

[19] Sivan, E., Spira, M., Achiron, R., et al. (2010) Prophylactic Pelvic Artery Catheterization and Embolization in Women with Placenta Accreta: Can It Prevent Cesarean Hysterectomy? American Journal of Perinatology, 27, 455-461.

[20] Alanis, M., Hurst, B.S., Marshburn, P.B. and Matthews, M.L. (2006) Conservative Management of Placenta Increta with Selective Arterial Embolization Preserves Future Fertility and Results in a Favorable Outcome in Subsequent Pregnancies. Fertility and Sterility, 86, 1514.e3-1514.

[21] Palacios-Jaraquemada, J.M. (2008) Diagnosis and Management of Placenta Accreta. Best Practice \& Research Clinical Obstetrics and Gynaecology, 22, 1133-1148. https://doi.org/10.1016/j.bpobgyn.2008.08.003

[22] Sentilhes, L., Goffinet, F. and Kayem, G. (2013) Management of Placenta Accreta. Acta Obstetricia et Gynecologica Scandinavica, 92, 1125-1134. https://doi.org/10.1111/aogs.12222

[23] Eller, A., Porter, T., Soisson, P. and Silver, R. (2009) Optimal Management Strategies for Placenta Accreta. BJOG, 116, 648-654. https://doi.org/10.1111/j.1471-0528.2008.02037.x 